\title{
Prevalêcia de sobrepeso e obesidade em individuos com HIV/AIDS: uma revisão integrativa
}

RESUMO | OBJETIVO: Conhecer a literatura acerca da prevalência de sobrepeso e obesidade em indivíduos vivendo com HIV/ AIDS. MÉTODO: Trata-se de uma revisão integrativa utilizando as bases de dados BVS, SciELO, LILACS, Science Direct, Cochrane Library e MEDLINE/PubMed segundo o instrumento pensado por Pompeo, Rossi e Galvão, com uso dos descritores em DeCS "sobrepeso", "obesidade", "hiv". Foram selecionadas publicações entre 2012 e 2020 em inglês, portugês e espanhol, com indivíduos HIV positivos. Foram excluídos estudos inconclusivos. RESULTADOS: Inicialmente foram encontrados 3029 estudos dos quais 18 atenderam aos critérios de inclusão. Há forte característica de aumento de peso a grande taxa de sedentarismo, grande prevalência de mulheres acima do peso, com baixa escolaridade e idade variando de 38 a 50 anos, raça negra, solteiros, baixa renda e com baixa escolaridade ainda faziam uso de drogas ilícitas. Essas características estão presentes em mais de 60\% dos pacientes com o fator clínico estudado. CONCLUSÃO: A introdução de medicamentos antirretrovirais no tratamento do HIV trouxe o aumento da prevalência de sobrepeso e obesidade entre os portadores de HIVIAIDS.

Palavras-chaves: Sobrepeso; Obesidade; HIV.

ABSTRACT I OBJECTIVE: To know the literature on the prevalence of overweight and obesity in individuals living with HIVIAIDS. METHOD: This is an integrative review using the databases VHL, SciELO, LILACS, Science Direct, Cochrane Library and MEDLINE/PubMed according to the instrument conceived by Pompeo, Rossi and Galvão, using the descriptors in DeCS "overweight", "obesity", "hiv". Publications were selected between 2012 and 2020 in English, Portuguese and Spanish, with HIV positive individuals. Inconclusive studies were excluded. RESULTS: Initially, 3029 studies were found, of which 18 met the inclusion criteria. There is a strong characteristic of weight gain at a high rate of sedentary lifestyle, a high prevalence of overweight women, with low schooling and age ranging from 38 to 50 years, black race, single, low income and with low schooling still used illicit drugs. These characteristics are present in more than $60 \%$ of patients with the clinical factor studied. CONCLUSION: The introduction of antiretroviral drugs in hiv treatment has led to an increase in the prevalence of overweight and obesity among HIV/AIDS patients.

Keywords: Overweight; Obesity; HIV.

RESUMEN | OBJETIVO: Conocer la literatura sobre la prevalencia del sobrepeso y la obesidad en las personas que viven con el VIH/ SIDA. METODO: Se trata de una revisión integradora utilizando las bases de datos VHL, SciELO, LILACS, Science Direct, Cochrane Library y MEDLINE/PubMed según el instrumento concebido por Pompeo, Rossi y Galvao, utilizando los descriptores de DeCS "sobrepeso", "obesidad", "hiv". Las publicaciones fueron seleccionadas entre 2012 y 2020 en inglés, portugués y español, con individuos seropositivos. Se excluyeron los estudios no concluyentes. RESULTADOS: Inicialmente, se encontraron 3029 estudios, de los cuales 18 cumplían con los criterios de inclusión. Hay una fuerte característica del aumento de peso a una alta tasa de estilo de vida sedentario, una alta prevalencia de mujeres con sobrepeso, con baja escolaridad y edad que van de 38 a 50 años, raza negra, soltero, de bajos ingresos y con baja escolarización todavía se utilizan drogas ilícitas. Estas características están presentes en más del $60 \%$ de los pacientes con el factor clínico estudiado. CONCLUSION: La introducción de medicamentos antirretrovirales en el tratamiento del vih ha dado lugar a un aumento de la prevalencia del sobrepeso y la obesidad entre los pacientes con VIH/SIDA. Palabras claves: Sobrepeso; Obesidad; VIH.

\section{Ednete Pereira da Silva}

Enfermeira graduada pelo Centro Universitário de Juazeiro do Norte - UNIJUAZEIRO. ORCID: 0000-0001-8124-2447

\section{Anderson Marcos Vieira do Nascimento \\ Mestrando em Saúde Pública pela Univer- sidade Estadual da Paraíba - UEPB. Enfer- meira graduada pelo Centro Universitário de Juazeiro do Norte - UNIJUAZEIRO. Membro do Grupo de Pesquisa e estudos em Ciências da Saúde cadastrado no Dire- tório do CNPq. \\ ORCID: 0000-0001-8575-1580}

Recebido em: 20/10/2020

Aprovado em: 16/11/2020

\section{José Rafael Eduardo Campos}

Graduando em Enfermagem pelo Centro Universitário de Juazeiro do Norte - UNIJUAZEIRO. Membro do Grupo de Pesquisa e estudos em Ciências da Saúde cadastrado no Diretório do CNPq e bolsista do Programa Institucional de Bolsas de Iniciação Científica (PIBIC).

ORCID: 0000-0003-0463-4839

\begin{tabular}{l}
\hline Jessika Brenda Rafael Campos \\
Enfermeira, Residente Multiprofissional \\
com ênfase em Transplante pelo Hospital \\
Universitário Walter Cantídio - UFC. Mem- \\
bro do Grupo de Pesquisa e estudos em \\
Ciências da Saúde cadastrado no Diretório \\
do CNPq. \\
ORCID: 0000-0003- 3768-5480
\end{tabular}

\section{Alyce Brito Barros}

Graduanda em Enfermagem pelo Centro Universitário de Juazeiro do Norte - UNIJUAZEIRO. Membro do Grupo de Pesquisa e estudos em Ciências da Saúde cadastrado no Diretório do CNPq.

ORCID: 0000-0002-2775-1264

\section{Dayse Christina Rodrigues Pereira Luz}

Pós-Doutoranda em Ciências da Saúde pelo Centro Universitário Saúdo do ABC- FMABC. Doutora em Ciências da Saúde pelo Centro Universitário Saúdo do ABC. Mestre em enfermagem pela Universidade Federal do Ceará - UFC. Docente do Centro Universitário de Juazeiro do Norte -UNIJUAZEIRO. Líder do Grupo de Pesquisa e estudos em Ciências da Saúde cadastrado no Diretório do CNPq. ORCID: 0000-0002-5719-3574 
INTRODUÇÃO

$\mathrm{O}$ Vírus da Imunodeficiência Humana (HIV) é transmitido por prática sexual desprotegido, compartilhamento de objetos perfurocortantes contaminados com sangue e por via vertical. Logo, após contaminada, a pessoa não desenvolve de imediato a Síndrome da Imunodeficiência Adquirida (AIDS) perpassando por diversas fases desde da fase aguda até a última da aids propriamente dita ${ }^{(1)}$.

A AIDS foi reconhecida em meados de 1981, nos Estados Unidos da América, a partir da identificação de um número elevado de pacientes adultos do sexo masculino e homossexuais, que apresentavam sarcoma de Kaposi, pneumonia por Pneumocystis carinii e comprometimento do sistema imune. Assim sendo, embora os primeiros óbitos atribuíveis a essa causa possam ter ocorrido nos anos 1950, acredita-se que a infecção tenha surgido nas regiões africanas central e oriental, uma vez que ali teve início sua maior frequência e onde a infecção de primatas ocorre na natureza ${ }^{(2)}$.

Atualmente o ganho de peso, a redistribuição de gordura e a obesidade são problemas nutricionais que os indivíduos com HIV/AIDS em uso da terapia estão apresentando. A marca do corpo, a imagem a ser cuidada e zelada, com o surgimento dos efeitos colaterais produzidos pelos inibidores como: Lipodistrofia e fatores coadjuvantes associados com o HIV/AIDS. Ademais, a comida não é apenas fonte de nutrição, mas está ligada a papéis relacionados a aspectos sociais, religiosos e econômicos ${ }^{(3)}$.

Antes a problemática estava concentrada em amenizar unicamente a questão da imunodeficiência e combate ao vírus com uso da terapia, mas agora já se tem a preocupação da segurança alimentar e nutricional como forma de minimizar os efeitos da obesidade por meio de diversos métodos (antropometria, consumo alimentar e exame físico). A qualidade da alimentação tornou-se um aspecto importante na vida dos pacientes como parte do tratamento.

Diante do exposto, questiona-se: Qual a prevalência de sobrepeso e obesidade em indivíduos vivendo com HIV/ AIDS? Seria a prevalência maior em muIheres ou em homens? E em que faixa etária são mais acometidas?

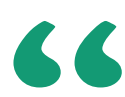

$$
\begin{gathered}
\text { A AIDS foi } \\
\text { reconhecida em } \\
\text { meados de 1981, } \\
\text { nos Estados Unidos } \\
\text { da América, } \\
\text { a partir da } \\
\text { identificação de um } \\
\text { número elevado de } \\
\text { pacientes adultos } \\
\text { do sexo masculino } \\
\text { e homossexuais, }
\end{gathered}
$$
que apresentavam sarcoma de Kaposi, pneumonia por Pneumocystis carinii e comprometimento do sistema imune.

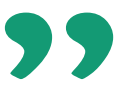

Além da segurança alimentar e nutricional para prevenção da obesidade, a Organização Mundial da Saúde adicionou à sua agenda, o incentivo a prática de atividades físicas. Para isso, a enfer- magem atua com grande importância no monitoramento dessa prática, sendo esta uma abordagem para acompanhar a atividade física ao longo do tempo, identificando também mudanças ${ }^{(1)}$.

A enfermagem constitui-se como campo de conhecimento aplicado na grande área da saúde e enquanto tal alinha-se às políticas de saúde de uma forma mais geral. Dessa forma, o alinhamento dos pesquisadores a essa realidade significa, não apenas aderir a uma determinada política, mas também potencializar esforços no sentido da resolução de alguns grandes problemas nacionais de saúde. Portanto, o objetivo da pesquisa foi analisar a literatura acerca da prevalência de sobrepeso e obesidade em indivíduos vivendo com HIV/AIDS.

\section{MÉTODO}

Trata-se de um estudo realizado por meio do método de revisão integrativa da literatura com abordagem quali-quantitativa.

O termo "integrativa" tem origem na integração de opiniões, conceitos ou ideias provenientes das pesquisas utilizadas. É nesse ponto que se evidencia o potencial para construir a ciência. Uma boa revisão integrativa contribui para o desenvolvimento de teorias. O método de revisão integrativa é uma abordagem que permite a inclusão de estudos que adotam diversas metodologias, ou seja, experimental e de pesquisa não experimental ${ }^{(4)}$.

O estudo ora proposto utilizou o modelo descrito por Mendes, Silveira e Galvão(5), o qual se dividiu em seis etapas: a) Primeira etapa: identificação do tema e seleção da hipótese para a elaboração da revisão integrativa; b) Segunda etapa: estabelecimento de critérios para inclusão e exclusão de estudos/ amostragem e busca na literatura; c) Terceira etapa: definição das informações a serem extraídas dos estudos selecionados/categorização dos estudos; d) Quarta etapa: avaliação dos estudos incluídos na revisão integrativa; 
e) Quinta etapa: interpretação dos resultados; f) Sexta etapa: apresentação da revisão/síntese do conhecimento.

A primeira etapa foi constituída na seleção da hipótese através da pergunta norteadora utilizando o método PICO referente ao problema, intervenção, comparação e desfecho. A pergunta foi norteada através do questionamento de prevalência de sobrepeso e obesidade em indivíduos vivendo com HIV/AIDS, se é maior em mulheres ou em homens e em que faixa etária são mais acometidos conhecendo a literatura sobre o assunto.

A segunda etapa se deu através do estabelecimento dos critérios de inclusão e exclusão a fim de nortear a busca e seleção dos artigos. Os critérios de inclusão foram artigos publicados de 2012 a 2020 em inglês, português e espanhol, visto a grande escassez de estudos sobre o assunto, participantes eram adultos ou crianças com diagnóstico de HIV / AIDS com qualquer desfecho. Foram excluídos estudos inconclusivos ou com avaliação metodológica com pouca evidência.

Os estudos foram selecionados por meio de busca eletrônica na Biblioteca Virtual em Saúde (BVS) e bases de dados Medical Literature Analysis and Retrie-

\section{Figura 1: Fluxograma de seleção dos estudos}

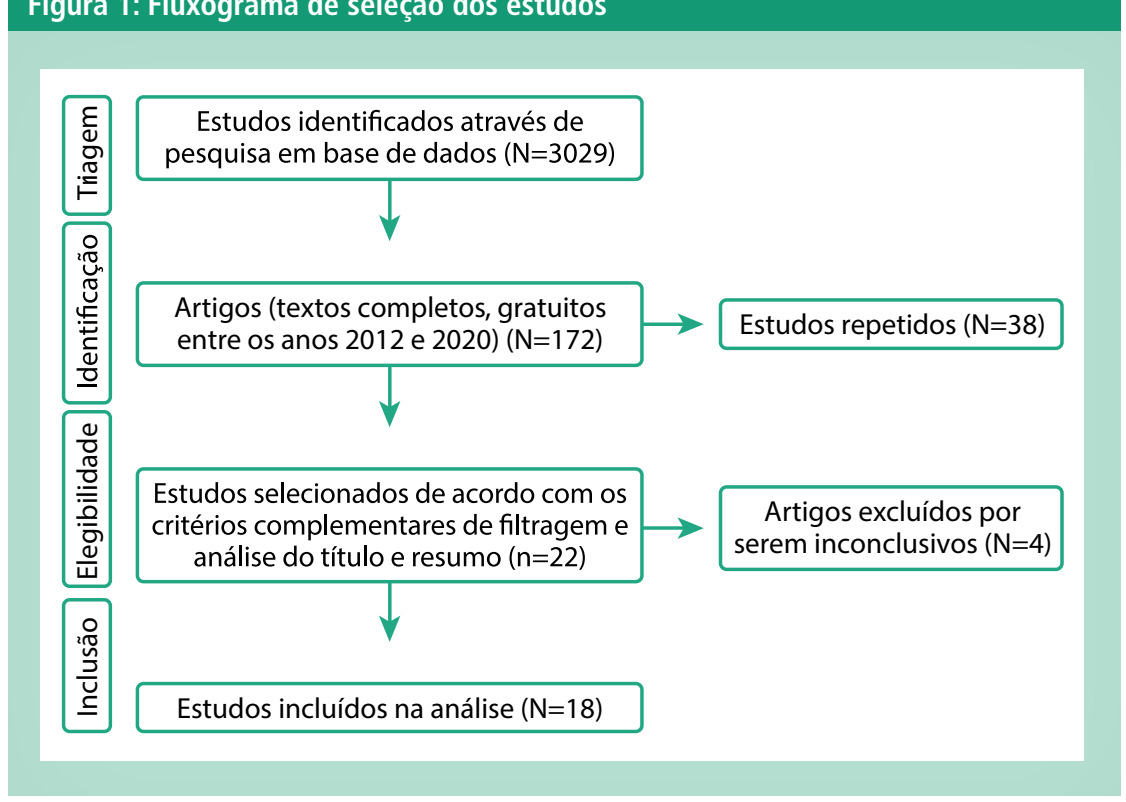

Fonte: Elaborado pelos autores (2020) val System Online (MEDLINE/PubMed), Literatura Latino-Americana e do Caribe em Ciências da Saúde (LILACS/BIREME) e Scientific Eletronic Library Online (SciELO), ScienceDirect e Cochrane Library, utilizando os descritores em DeCS: "sobrepeso", "obesidade" e "HIV", com uso do operador Booleano AND.

A terceira etapa foi constituída da categorização dos resultados através da composição de um quadro composto por Autor/ ano/Base de dados, objetivos, desfecho, confiabilidade e qualidade (média), nível de evidência, uma tabela com características relativas aos anos, base de dados, idiomas das publicações e outra tabela com o perfil dos pacientes com sobrepeso e obesidade vivendo com HIV/ AIDS.

A quarta etapa deu-se através da classificação do nível de evidência dos artigos, proposto por Pompeo, Rossi e Galvão ${ }^{(6)}$, do nível de melhor qualidade metodológica ao nível de menor evidência que foram os seguintes:

- Nível I de evidência - Revisão sistemática ou metanálise (síntese das evidências de todos os relevantes ensaios clínicos randomizados). 
Quadro 1: Características do estudo

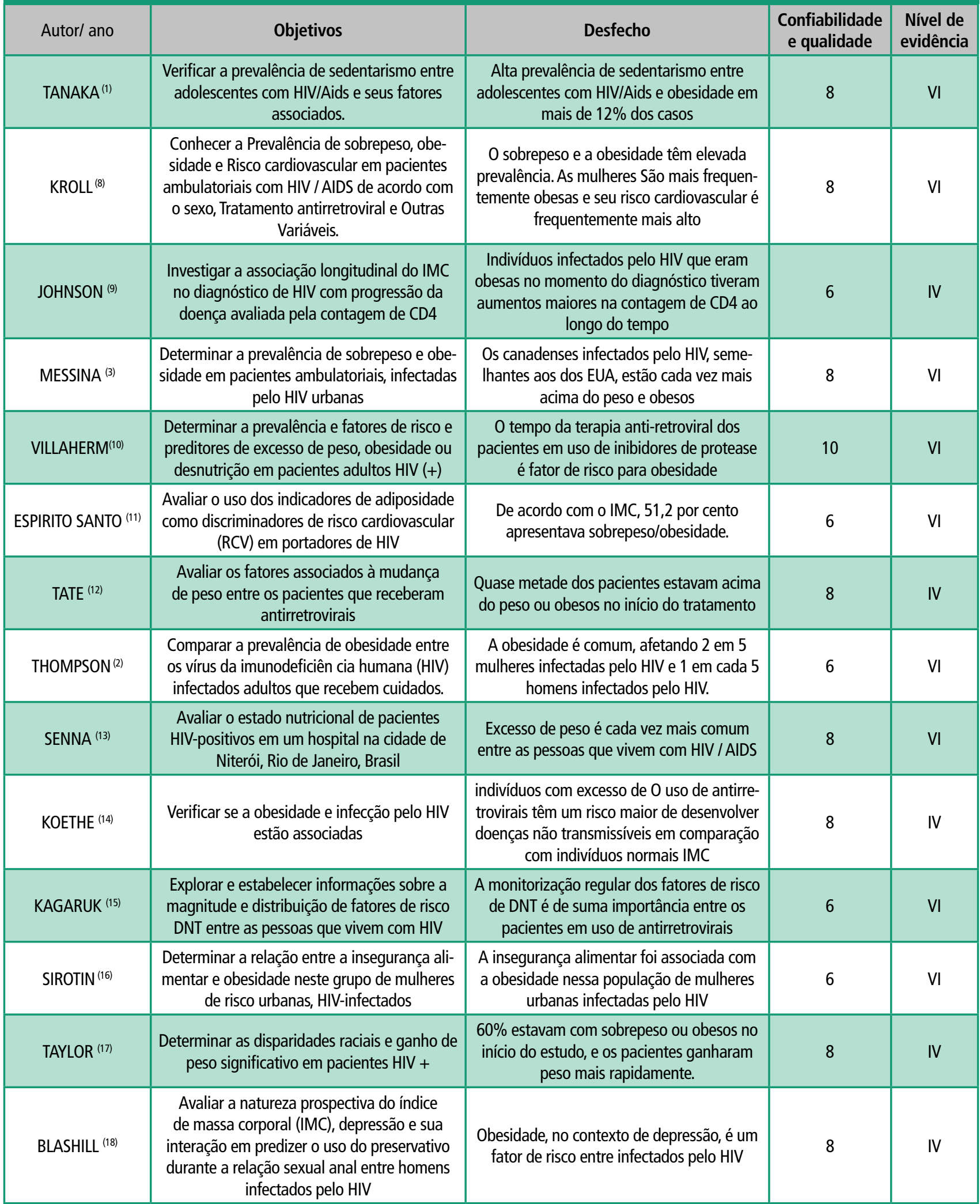




\begin{tabular}{|c|c|c|c|c|}
\hline $\begin{array}{c}\text { KOETHE; HUL- } \\
\text { GAN; NISWEN- } \\
\text { DER }{ }^{(19)}\end{array}$ & Verificar a relação entre HIV e obesidade & $\begin{array}{c}\text { Como a proporção de indivíduos infectados pelo HIV } \\
\text { com sobrepeso obesidade continua a aumentar, a } \\
\text { compreensão do papel do tecido adiposo na patogêne- } \\
\text { se da ativação imune será fundamental }\end{array}$ & 6 & V \\
\hline $\begin{array}{c}\text { WAND; RAMJEE } \\
\text { (20) }\end{array}$ & $\begin{array}{c}\text { Descrever a prevalência de obesidade em uma } \\
\text { coorte de mulheres sul-Africano e discutir as impli- } \\
\text { cações para as práticas de saúde pública. }\end{array}$ & $\begin{array}{c}\text { Aproximadamente } 70 \% \text { da população da amostra } \\
\text { foram classificadas como sendo excesso de peso ou } \\
\text { obesos. }\end{array}$ & 8 & III \\
\hline LAKEY et al ${ }^{(21)}$ & Verificar relação entre ganho de peso e HIV & $\begin{array}{c}\text { As altas taxas de sobrepeso / obesidade em pessoas } \\
\text { infectadas pelo HIV }\end{array}$ & 10 & IV \\
\hline $\begin{array}{c}\text { POURCHER; } \\
\text { COSTAGLIOLA; } \\
\text { MARTINEZ }{ }^{(22)}\end{array}$ & Descrever a prevalência de obesidade no HIV & $\begin{array}{c}\text { A prevalência da obesidade é de } 15,1 \% \text { em mulheres e } \\
5,3 \% \text { em homens. }\end{array}$ & 8 & VI \\
\hline Fonte: Elaborado pelos autores & & & \\
\hline
\end{tabular}

Tabela 1: Demonstração em tabela da quantidade de artigos disposta em cada base de dados e em que idiomas se encontravam.

Característica do estudo Número encontrado \% em relação ao total Idiomas das publicações

\begin{tabular}{lcc} 
Inglês & 15 & 83,3 \\
Português & 3 & 16,7 \\
\hline Base de dados & & \\
PubMed/MEDLINE & 12 & 66,7 \\
SciELO & 2 & 11,1 \\
LILACS & 2 & 11,1 \\
Science Direct & 2 & 11,1 \\
Cochrane Library & 0 & 0 \\
\hline TOTAL & 18 & 100
\end{tabular}

Fonte: Elaborado pelos autores (2020)
A tabela 1, seguinte, evidencia em quais bases de dados estavam hospedados os artigos e os idiomas os quais eles estavam escritos.

A seguir, a tabela 2 aponta algumas características descritas pelos autores que os pacientes analisados possuíam. A ordem das características do perfil foi retirada seguindo a ordem de estudos de cima para baixo apresentados no quadro 1 .

\section{DISCUSSÃO}

A maioria dos autores determinou conhecer a prevalência de sobrepeso e obesidade entre pacientes que tinham HIV/AIDS.

\section{Tabela 2: Perfil dos pacientes}

Perfil mais prevalente dos pacientes com sobrepeso e obesidade vivendo com HIVIAIDS

Pouca atividade física, idade de 10 a 19 anos, proporção maior de meninas, diagnóstico de HIV de 0 a 2 anos, terapia antirretroviral tripla, HDL colesterol alterado, renda >= a 1 salário mínimo

Maior parte do sexo feminino, muita prevalência de risco cardiovascular, baixo número de células CD4

Idade acima de 18 anos, taxas menores de CD4 para pessoas mais obesas, $60 \%$ masculino, raça negra, solteiros, ensino médio completo

$90 \%$ masculino, 35\% já usaram drogas injetáveis e ilícitas, raça negra mais prevalente a obesidade

0 uso do tempo da terapia anti-retroviral que os pacientes têm de se relacionar 0 uso de inibidores de protease como um fator de risco

Média de 35 anos, parâmetros aumentados de razão cintura/quadril (RCQ) e da razão cintura/estatura (RCEst)

Maior parte homens, IMC elevado, alteração de adiposidade em média 24 meses depois de início do tratamento
Número de pacientes com a característica (exceto sexo)

Porcentagem referente ao número de pacientes (\%) 


\begin{tabular}{lcc}
$\begin{array}{l}\text { Maior parte homens, IMC elevado, alteração de adiposidade em média } 24 \text { meses } \\
\text { depois de início do tratamento }\end{array}$ & 681 & 1.02 \\
$\begin{array}{l}\text { Idade entre } 40 \text { e } 60 \text { anos, CD4 }<200 \text { cél/ml, baixa escolaridade, raça negra, } \\
\text { baixa renda }\end{array}$ & 4006 & 6.03 \\
\hline $\begin{array}{l}\text { Maior proporção de mulheres, idade de } 41 \text { a } 50 \text { anos } \\
\text { IMC elevado, uso de antirretroviral acima de } 2 \text { anos, alta prevalência de sobre- } \\
\text { peso e obesidade }\end{array}$ & 235 & 0.35 \\
\hline $\begin{array}{l}\text { Colesterol total aumentado, grande prevalência de diabetes e hipertensão, idade } \\
>\text { de } 40 \text { anos }\end{array}$ & 1089 & 1.64 \\
\hline Uso de álcool e outras drogas, maioria negros & 754 & 0.35 \\
\hline Maioria brancos, femininas, escolaridade média e baixa renda & 231 & 1.83 \\
\hline Obesidade e depressão com altas taxas de prevalência & 1214 & 0.74 \\
\hline Brancos, alto peso & 490 & 1.83 \\
\hline Idades > 35 anos, IMC superior a 25 & 1214 & 8.27 \\
\hline Média de idade de 38 anos, sexo feminino prevalente & 5495 & 0.14 \\
\hline Sedentarismo, mulheres, altas taxas de obesidades e comorbidades associadas. & 92 & 73.56 \\
\hline TOTAL & 48897 & 100 \\
\hline
\end{tabular}

Fonte: Elaborado pelos autores (2020)

Os autores como Kroll ${ }^{(8)}$, Johnson ${ }^{(9)}$, Thompson-Paul ${ }^{(2)}$, Sirotin ${ }^{(16)}$ e Pourcher; Costagliola e Martinez ${ }^{(22)}$ compartiIham resultados semelhantes no que se refere a maior prevalência de sobrepeso e obesidade associado ao HIV de maneira mais prevalente entre mulheres. Além disso, doenças cardiovasculares e baixas taxas de CD4 em períodos de pico de peso são normalmente encontradas nas pacientes.

Essa alta taxa de obesidade não é só mais prevalente na vida adulta. Adolescentes infectados pelo HIV também demonstraram altos índices de IMC podendo identificar uma das origens da obesidade na vida adulta. Esse fato é normalmente resultante de taxas elevadas de sedentarismo verificada na população que quando associado a outros fatores potencializam a chance de aumento de peso ${ }^{(1)}$.

Outros autores como Villahermosa ${ }^{(10)}$, Koethe ${ }^{(14)}$, Kagaruki ${ }^{(15)}$ e Lakey ${ }^{(21)} \mathrm{com}$ partilham a premissa que fatores não totalmente conhecidos relacionados a medicação em uso podem predispor o indivíduo ao aumento de peso. A hipótese mais prevalente versa sobre a influ-

\section{0}

Essa alta taxa de obesidade não é só mais prevalente na vida adulta. Adolescentes infectados pelo HIV também demonstraram altos índices de IMC podendo identificar uma das origens da obesidade na vida adulta. ência dos antirretrovirais com o metabolismo do tecido adiposo aumentando sua formação principalmente na região abdominal.

Kroll ${ }^{\left({ }^{8)}\right.}$, Senna ${ }^{(13)}$, Taylor ${ }^{(17)}$ e Lakey ${ }^{(21)}$ encontraram grande prevalência de muIheres acima do peso, com baixa escolaridade e idade variando de 38 a 50 anos. Essas características normalmente condizem com o perfil de pacientes com HIV e acima do peso. Esses fatores são associados principalmente a baixos níveis de células CD4 e características genéticas associadas.

Em contrapartida, os autores Johnson $^{(9)}$, Messina; Mccall e Barron ${ }^{(3)}$, Thompson-Paul (2) e Tate ${ }^{(12)}$ encontraram mais prevalente pessoas do sexo masculino, raça negra, solteiros, baixa renda e com baixa escolaridade. Essas características podem estar associadas com a amostra selecionada, mas é um aspecto que deve ser levado em consideração.

Os inibidores de protease é a classe de medicamentos que está mais fortemente associado ao aumento de peso nos usuários. Apesar dos fatores bioquímicos envolvidos não estarem to- 
talmente esclarecidos a devida atenção deve ser dada para uma atenção à saúde mais integral ${ }^{(10)}$.

Sirotin ${ }^{(16)}$, Messina; Mccall E Barron $^{(3)}$ encontraram que grande parte dos pacientes também já fizeram ou ainda faziam uso de drogas ilícitas. Esse fato pode alterar determinadas propriedades e ação correta das medicações predispondo a alguma alteração do peso.

Espírito Santo ${ }^{(11)}$, Koethe ${ }^{(14)}$, Kagaruki ${ }^{(15)}$, Wand; Ramjee ${ }^{(20)}$ compartilham os achados antropométricos elevados tais como circunferência da cintura, IMC, comorbidades associadas como hipertensão além do tempo de diagnóstico e antirretrovirais acima de dois anos. Apenas o autor Blashill ${ }^{(18)}$ encontrou transtorno mental (depressão) associado ao HIV e obesidade conjuntamente. Focar em outras características clínicas além da principal é de larga importância para um correto manejo terapêutico.

\section{CONCLUSÃO}

A introdução de medicamentos antirretrovirais no tratamento do HIV trou- xe grande melhora no padrão de saúde das pessoas acometidas. Porém outra característica relacionada à medicação foi o aumento da prevalência de sobrepeso e obesidade entre os portadores de HIV/AIDS.

Os pacientes compartilham características como serem na maioria solteiros, baixas taxas de CD4, serem do sexo feminino, serem normalmente sedentários, possuírem baixas taxas de escolaridade e renda. Essas características estão presentes em mais de $60 \%$ dos pacientes com o fator clínico estudado.

\section{Referências}

1. Tanaka LF, Latorre MRDO, Silva AM, Konstantyner TCRO, Peres SV, Marques HHS. Alta prevalência de sedentarismo em adolescentes que vivem com HIV/Aids. Revista Paulista de Pediatria, v. 33, (n. 3), p. 326-331, set. 2015. Doi: https://doi.org/10.1016/j.rpped.2014.12.003

2. Thompson-Paul AM, et al. Obesity Among HIV-Infected Adults Receiving Medical Care in the United States: Data From the Cross-Sectional Medical Monitoring Project and National Health and Nutrition Examination Survey. Medicine, v. 94, (n. 27), p. e1081, jul. 2015. Doi: 10.1097 / MD.0000000000001081

3. Messina J, Mccall J, Barron A. Overweight and obesity status in an urban Canadian HIV outpatient population. The Journal of the Association of Nurses in AIDS Care : JANAC, v. 25, (n. 6), p. 652-6, jan. 2014. Doi: 10.1016/j.jana.2014.04.002

4. Botelho LLR, Cunha CCA, Macedo M. Método da revisão integrativa nos estudos organizacionais. Gestão e Sociedade - Belo Horizonte, maio/ agosto. v. 5, (n. 11), p. 121-136, 2011. DOI: https://doi.org/10.21171/ges. v5i11.1220

5. Mendes KDS, Silveira RCC, Galvão C. Revisão integrativa: método de pesquisa para a incorporação de evidencias na saúde e na enfermagem. Texto Contexto Efer, Florianópolis; v. 17, (n.4), p. 758-64. Out-Dez, 2008 6. Pompeo DA, Rossi LA, Galvão, CM. Revisão integrativa: etapa inicial do processo de validação de diagnóstico de enfermagem. Acta Paul Enferm; v. 22, (n.4), p. 434-8, 2009.

7Keynes Milton Primary Care Trust. Critical Appraisal Skills Programme (CASP). Making sense of evidence. London (UK): Oxford; 2002.

8. Kroll AF, Sprinz E, Leal SC, Labréa Mda L, Setúbal S. Prevalence of obesity and cardiovascular risk in patients with HIVIAIDS in Porto Alegre, Brazil. Arquivos Brasileiros de Endocrinologia \& Metabologia, v. 56, (n. 2), p. 137-141, 2012.

9. Johnson KD, Cai B, Duffus W. Longitudinal association between BMI at diagnosis and HIV disease progression. AIDS and behavior, v. 18, (n. 11), p. 2249-57, nov. 2014. https://doi.org/10.1007/s10461-014-0805-0 10. Villahermosa ML. Risk factors for overweith, obesity and malnutrition in HIV positive attending nutritional consultation center in patiens with infectious diseases Faculty of Dentistry. Acta Odontol Venez, 2013.

11. Espirito Santo R. Evaluation of cardiovascular risk through indicators of obesity and body fat distribution in hiv haart-naïve patients. p. $x v, 76$, 2013.

12. Tate $\mathrm{T}$, et al. HIV infection and obesity: where did all the wasting go? Antiviral therapy, v. 17, (n. 7), p. 1281-9, jan. 2012. Doi: 10.3851 / IMP2348
13. Senna AFK, Oliveira SA, Velarde LGC, Setúbal S. Nutritional status of HIV-positive patients in Niterói, Rio de Janeiro, Brazil. Journal of health, population, and nutrition, v. 32, (n. 4), p. 595-9, dez. 2014.

14. Koethe JR, Hulgan T, Niswender K. Adipose tissue and immune function: a review of evidence relevant to HIV infection. The Journal of infectious diseases, v. 208, (n. 8), p. 1194-201, 15 out. 2013. Doi: 10.1093/ infdis/jit324

15. Kagaruki GB, et al. Magnitude and risk factors of non-communicable diseases among people living with HIV in Tanzania: a cross sectional study from Mbeya and Dar es Salaam regions. BMC public health, v. 14, p. 904, jan. 2014. Doi: 10.1186 / 1471-2458-14-904

16. Sirontin N, Hoover DR, Shi Q, Anastos K, Weiser SD. Food insecurity with hunger is associated with obesity among HIV-infected and at risk women in Bronx, NY. PloS one, v. 9, (n. 8), p. e105957, jan. 2014. Doi: 10.1371 / journal.pone. 0105957

17. Taylor BS, Liang Y, Garduno S, Walter E. High risk of obesity and weight gain for HIV-infected uninsured minorities. Journal of acquired immune deficiency syndromes (1999), v. 65, (n. 2), p. e33-40, 1 fev. 2014. DOI: 10.1097 / QAl.0000000000000010

18. Blashill AJ, et al. Body mass index, depression, and condom use among HIV-infected men who have sex with men: a longitudinal moderation analysis. Archives of sexual behavior, v. 43, (n. 4), p. 729-34, maio 2014. Doi: 10.1007 / s10508-013-0155-4

19. Koethe JR, Jenkins CA, Bebawy S, Pastor S, Wester CW, Sterling TR. Body mass index and the risk of incident noncommunicable diseases after starting antiretroviral therapy. HIV medicine, v. 16, (n. 1), p. 67-72, jan. 2015. Doi: https://doi.org/10.1111/hiv.12178

20. Wand $H$, Ramjee G. High prevalence of obesity among women who enrolled in HIV prevention trials in KwaZulu-Natal, South Africa: healthy diet and life style messages should be integrated into HIV prevention programs. BMC public health, v. 13, p. 159, jan. 2013. Doi: $10.1186 /$ 1471-2458-13-159

21. Lakey W, Yang LY, Yancy W, Chow SC, Hicks C. Short communication: from wasting to obesity: initial antiretroviral therapy and weight gain in HIV-infected persons. AIDS research and human retroviruses, v. 29, (n. 3), p. 435-40, mar. 2013. Doi: 10.1089 / aid.2012.0234

22. Pourcher G, Costagliola D, Martinez, V. Obesity in HIV-infected patients in France: prevalence and surgical treatment options. Journal of visceral surgery, v. 152, (n. 1), p. 33-37, 2015. Doi: https://doi.org/10.1016/j.jviscsurg.2014.12.001. 